\title{
PROBLEMS OF CLOSEST APPROXIMATION CONNECTED WITH THE SOLUTION OF LINEAR DIFFERENTIAL EQUATIONS*
}

\author{
BY
}

W. H. McEWEN

1. Introduction. Consider the differential system consisting of the $m$ thorder ordinary linear differential equation

$$
L(y) \equiv \frac{d^{m} y}{d x^{m}}+Q_{1}(x) \frac{d^{m-1} y}{d x^{m-1}}+\cdots+Q_{m}(x) y=R(x),
$$

in which the functions $Q_{1}(x), \cdots, Q_{m}(x), R(x)$ are defined and continuous on $a \leqq x \leqq b$, and the $m$ linearly independent two-point boundary conditions

$$
U_{i}(y) \equiv \sum_{j=1}^{m}\left\{\alpha_{i}^{(i-1)} y^{(j-1)}(a)+\beta_{i}^{(j-1)} y^{(j-1)}(b)\right\}=h_{i} \quad(i=1,2, \cdots, m) .
$$

Let it be assumed that the system has a unique solution $y(x)$, or in other words that the corresponding reduced system is incompatible. Let $\phi_{1}(x), \phi_{2}(x), \ldots$ be an infinite sequence of functions, defined and continuous and linearly independent (in finite subsets) and having continuous derivatives of all orders up to and including the $m$ th on the interval, and let

$$
y_{n}(x)=c_{1} \phi_{1}(x)+c_{2} \phi_{2}(x)+\cdots+c_{n} \phi_{n}(x)
$$

be a linear combination of the first $n$ of these functions, subject to the restriction that $y_{n}(x)$ shall satisfy the boundary conditions, but otherwise arbitrary. Then it is a problem of minima to determine a sum of this type for which the integral $\int_{a}^{b}\left|L\left(y_{n}\right)-R\right| r d x$ is as small as possible, where $r$ is any given positive real number. Such a function, when it exists, will be called "a minimizing function of $\operatorname{order} n . "$

The aim of this paper is to investigate the questions of the existence of such a function and the convergence of it and certain of its derivatives as $n$ becomes infinite, the convergence problem, however, being treated only for the cases when $y_{n}(x)$ is a trigonometric sum or a polynomial. Kryloff $\dagger$ and

* Presented to the Society, September 12, 1930; received by the editors June 6, 1931.

† See, for example, N. Kryloff, Sur une méthode, basée sur le principe de minimum, pour l'intégration approchée des équations différentielles, Comptes Rendus, vol. 181 (1925), pp. 86-88. This is just one of a long series of papers by Kryloff dealing with more or less closely related problems; see also a volume by him in the Mémorial des Sciences Mathématiques, fascicule 49, Les Méthodes de Solution A pprochée des Problèmes de la Physique Mathématique, Paris, Gauthier-Villars, 1931. 
Krawtchouk* have considered the convergence question for a second-order system with the simple boundary conditions $y(a)=y(b)=0$, but only for the case when $r=2$. We shall treat the problem in its more general aspect by methods which are essentially different from those of the authors cited. In this connection mention should be made also of a paper by Picone $†$ which deals with a different but somewhat analogous problem of minima, relating mainly to a second-order differential system, but involving an arbitrary power of the error.

2. Existence of a minimizing function. Uniqueness. In discussing the question of existence we must distinguish three cases in respect to the boundary conditions:

(a) when they are homogeneous and are satisfied by the $\phi$ 's individually,

(b) when they are homogeneous but are not satisfied by the $\phi$ 's individually,

(c) when they are non-homogeneous.

The first case can be disposed of immediately. When the boundary conditions are homogeneous and are satisfied by the $\phi$ 's individually the problem reduces to that of approximating $R(x)$ by means of a linear combination of the $n$ functions $L\left(\phi_{1}\right), L\left(\phi_{2}\right), \cdots, L\left(\phi_{n}\right)$ to give the best approximation in the sense of the method of least $r$ th powers, and it is well known that this problem has a solution when $r>0$, and indeed a unique solution when $r>1$ and the functions $L\left(\phi_{1}\right), \cdots, L\left(\phi_{n}\right)$ are linearly independent. $\begin{gathered}\text { This latter } \\ \text {. }\end{gathered}$ condition, of course, means that the homogeneous differential equation $L(y)=0$ has no non-trivial solution which is a linear combination of $\phi_{1}, \cdots, \phi_{n}$, and this requirement in turn is met, by reason of the boundary conditions satisfied by the $\phi$ 's, if the homogeneous system $L(y)=0, U_{i}(y)=0$, $i=1,2, \cdots, m$, is incompatible.

When, on the other hand, the boundary conditions are non-homogeneous, or are homogeneous but are not satisfied by the $\phi$ 's individually, the additional question arises whether it is possible to satisfy these conditions by sums of the form $y_{n}(x)$ at all. The requirement is that the $n$ coefficients $c_{k}$ satisfy the $m$ linear equations

$$
\sum_{k=1}^{n} c_{k} U_{b}\left(\phi_{k}\right)=h_{i} \quad(i=1,2, \cdots, m)
$$

\footnotetext{
* See, for example, M. Krawtchouk, Sur les dérivées des intégrales approchées de certaines équations différentielles, Rendiconti del Circolo Matematico di Palermo, vol. 54 (1930), pp. 194-198.

$\dagger$ M. Picone, Sul metodo delle minime potenze ponderate e sul metodo di Ritz, etc., Rendiconti del Circolo Matematico di Palermo, vol. 52 (1928), pp. 225-253.

$\ddagger$ For a proof, see, for example, D. Jackson, On functions of closest approximation, these Transactions, vol. 22 (1921), pp. 117-128, pp. 118-122; A generalized problem in weighted approximation, these Transactions, vol. 26 (1924), pp. 133-154; pp. 133-138.
} 
If the numbers $h_{i}$ are all zero there will certainly be infinitely many sets of the c's satisfying these equations for values of $n>m$. If they are not all zero, but if the equations can be satisfied for a particular value of $n$, then they can be satisfied by infinitely many choices of the $c$ 's for larger values of $n$, since the sum of a solution of the non-homogeneous equations and an arbitrary solution of the homogeneous equations will satisfy the non-homogeneous equations. Incidentally, it will be shown in $\$ 3$ (in connection with the proof of Theorem $\mathrm{E}$ ) that this condition can actually be met in the one case of immediate importance, that of polynomials.

Let it be assumed that linear combinations of $\phi_{1}, \cdots, \phi_{n}$ satisfying the boundary conditions do exist; it is assumed also, as already stated, that the reduced system $L(y)=0, U_{i}(y)=0, i=1,2, \cdots, m$, is incompatible. Then, in particular, a linear combination of the $\phi$ 's satisfying the homogeneous boundary conditions will not satisfy the reduced differential equation unless it vanishes identically.

With the qualifications just cited the minimizing problem in cases (b) and (c) also has a solution when $r>0$, and a unique solution when $r>1$. If the functions $L\left(\phi_{1}\right), \cdots, L\left(\phi_{n}\right)$ are linearly independent, the existence of a solution can be inferred almost immediately from the second paper referred to in the last footnote. For the essential part of the argument there depended on showing that the $c$ 's for which the integral to be minimized has values not greater than a specified upper bound are to be sought in a closed region of $n$-dimensional space; and the addition of the auxiliary conditions has the effect merely of narrowing the consideration to a subset of this closed region, which is likewise closed. If the equation $L(y)=0$, while not satisfied by any non-trivial combination of the $\phi$ 's subject to the boundary conditions, is satisfied by other linear combinations of the $\phi$ 's, the argument has to be re-examined with a little more care. In the case of homogeneous boundary conditions all linear combinations of the $\phi$ 's satisfying the conditions can be expressed linearly in terms of a fundamental system of such combinations, and the proof can be carried through for approximation in terms of the functions of this fundamental system. For the case of non-homogeneous conditions it is to be noted that if $\Phi$ is a particular combination of the $\phi$ 's satisfying the conditions the problem of approximating $R(x)$ by a combination satisfying the non-homogeneous conditions is the same as that of approximating $R-L(\Phi)$ by a combination satisfying the corresponding homogeneous conditions.

As to the uniqueness proof, the fact that the arithmetical average of two solutions of the auxiliary conditions is itself a solution, even in the non- 
homogeneous case, is sufficient to insure the applicability of the method used in the passage to which reference has been made.

3. Preliminary theorems on approximation. In this section we shall introduce certain theorems on approximation which in part at least are well known in substance, if not in all cases in the exact form in which they are stated here. The letter $m$ will be used throughout the section to denote any arbitrarily chosen positive integer, not necessarily the order of the differential system, although in the applications to be made later the particular $m$ of the differential system will be the one required. The first theorem is an extension of the Weierstrass theorem for trigonometric sums:

THEOREM A. If $f(x)$ is a given function which is continuous and periodic with the period $2 \pi$, and if $f^{\prime}(x), \cdots, f^{(m)}(x)$ exist and are continuous, then for any given positive number $\epsilon$ there exists a trigonometric sum $T_{n}(x)$ of some order $n$ such that

$$
\left|f^{(k)}(x)-T_{n}^{(k)}(x)\right| \leqq \epsilon,
$$

for $k=0,1, \cdots, m$, and for all values of $x$.

The truth of this statement is apparent at once from a consideration of the Fejér mean

$$
\sigma_{n}(x)=\frac{1}{n \pi} \int_{-\pi}^{\pi} f(\xi) \phi(\xi-x) d \xi,
$$

where

$$
\phi(\xi-x)=\frac{\sin ^{2}\left(\frac{n}{2}(\xi-x)\right)}{2 \sin ^{2}\left(\frac{1}{2}(\xi-x)\right)} .
$$

It is well known that this function, which is a trigonometric sum of order $n-1$ in $x$, converges uniformly to $f(x)$ as $n$ becomes infinite. Hence by taking $n$ sufficiently large it can be made to approximate $f(x)$ with an error less than any preassigned positive number $\epsilon$. If this function is differentiated with respect to $x$ and $\partial \phi / \partial x$ replaced by its equal $-\partial \phi / \partial \xi$ and the resulting expression integrated by parts, it will be seen that

$$
\sigma_{n}{ }^{\prime}(x)=\frac{1}{n \pi} \int_{-\pi}^{\pi} f^{\prime}(\xi) \phi(\xi-x) d \xi .
$$

But this is the Fejér mean of $f^{\prime}(x)$ and it therefore converges to $f^{\prime}(x)$ as $n$ becomes infinite. By repetitions of this process it can be shown that the higher derivatives of $\sigma_{n}(x)$ converge to the respective derivatives of $f(x)$. Hence the theorem is established. 
The extended form of the Weierstrass theorem for polynomials we shall call

THEOREM B. If $f(x)$ is a given function which is defined and continuous on $a \leqq x \leqq b$, and if $f^{\prime}(x), \cdots, f^{(m)}(x)$ exist and are continuous there, then for any given positive number $\epsilon$ there exists a polynomial $P_{n}(x)$ of some degree $n$ such that $\left|f^{(k)}(x)-P_{n}^{(k)}(x)\right| \leqq \epsilon$, for $k=0,1, \cdots, m$, and for all values of $x$ on the interval.

Consider the function $f^{(m)}(x)$. It is continuous on $(a, b)$, and therefore by Weierstrass' theorem a polynomial $Q_{1}(x)$ of some degree $N$ can be found, such that

$$
\left|f^{(m)}(x)-Q_{1}(x)\right| \leqq \eta, a \leqq x \leqq b,
$$

where $\eta$ is any preassigned positive number. Let

Then

$$
Q_{2}(x)=f^{(m-1)}(a)+\int_{a}^{x} Q_{1}(x) d x .
$$

$$
\left|f^{(m-1)}(x)-Q_{2}(x)\right|=\left|\int_{a}^{x}\left[f^{(m)}(x)-Q_{1}(x)\right] d x\right| \leqq(b-a) \eta .
$$

By successive integrations, with suitable determination of the constant of integration at each stage, polynomials $Q_{3}(x), \cdots, Q_{m+1}(x)$ can be obtained, of degrees $N+2, \cdots, N+m$, respectively, so that each is the derivative of the following, and so that

$$
\left|f^{(m-2)}(x)-Q_{3}(x)\right| \leqq(b-a)^{2} \eta, \cdots,\left|f(x)-Q_{m+1}(x)\right| \leqq(b-a)^{m} \eta .
$$

The positive number $\epsilon$ being given, let $\eta=\epsilon$ or $\epsilon /(b-a)^{m}$, according as $b-a$ is $\leqq 1$ or $>1$, and let $P_{n}(x)=Q_{m+1}(x)$. Then $P_{n}(x)$ is a polynomial of degree $n=N+m$, and $\left|f^{(k)}(x)-P_{n}^{(k)}(x)\right| \leqq \epsilon$ for $k=0,1, \cdots, m$.

For the discussion of rapidity of convergence we shall need to know what degree of approximation can be obtained for a specified value of $n$. In this connection we have

Theorem C. If, in addition to the hypotheses of Theorem $\mathrm{A}$, it is assumed that $f^{(m)}(x)$ satisfies a Lipschitz condition

$$
\left|f^{(m)}\left(x_{1}\right)-f^{(m)}\left(x_{2}\right)\right| \leqq \lambda\left|x_{1}-x_{2}\right|,
$$

then for each positive integral value of $n$ there exists a trigonometric sum $T_{n}(x)$ of the nth order such that $\left|f^{(k)}(x)-T_{n}^{(k)}(x)\right| \leqq A / n$ for $k=0,1, \cdots, m$, and for all values of $x$, where $A$ is a constant independent of $n$. 
Under the hypothesis that $f^{(m)}(x)$ satisfies a Lipschitz condition it is possible to find for each positive integral value of $n$ a trigonometric sum $T_{n}(x)$ of order $n$ such that

$$
\left|f(x)-T_{n}(x)\right| \leqq \frac{K_{m+1} \lambda}{n^{m+1}},
$$

where $K_{m+1}$ is a constant independent of $n{ }^{*}$ Such a sum, for example, is the function $\dagger$

$$
\begin{aligned}
I_{s}(x)=h_{s} & \int_{-\pi / 2}^{\pi / 2}\left[ \pm f(x+2(m+1) u) \mp\left(\begin{array}{c}
m+1 \\
1
\end{array}\right) f(x+2 m u)+\cdots\right. \\
& \left.+\left(\begin{array}{c}
m+1 \\
m
\end{array}\right) f(x+2 u)\right]\left[\frac{\sin s u}{s \sin u}\right]^{2 \kappa} d u,
\end{aligned}
$$

in which the number $\kappa$ is the smallest integer such that $2 \kappa-(m+1)>1, h_{s}$ satisfies the equation

$$
\frac{1}{h_{s}}=\int_{-\pi / 2}^{\pi / 2}\left[\frac{\sin s u}{s \sin u}\right]^{2 \kappa} d u
$$

and $s$ bears a determinate relationship to $n$. The discussion here will be based on the properties of the function $I_{s}(x)$, as set forth in the passage cited, rather than on the somewhat different treatment in the Colloquium.

Although the form of $I_{s}(x)$ has to be readjusted if it is desired to pass from a given value of $m$ to a higher value, the form which corresponds to any given $m$ can be used to approximate $f(x)$ with a lower order of approximation than $1 / n^{m+1}$ when it happens that $f(x)$ exhibits a correspondingly lower order of continuity. For example, if $f^{(m-1)}(x)$ satisfies a Lipschitz condition the $I_{s}(x)$ corresponding to the value $m$ will approximate $f(x)$ with an error not greater than a constant multiple of $1 / n^{m}$.

By writing this integral as the sum of the $m+1$ integrals corresponding to the $m+1$ terms in the first factor of the integrand, suitably changing the variable of integration and the limits of integration in each part, and recombining again into a single integral, we obtain an expression of the form

$$
I_{s}(x)=h_{s} \int_{-\pi}^{\pi} f(\xi) \Phi(\xi-x) d \xi
$$

* D. Jackson, The Theory of Approximation, American Mathematical Society Colloquium Publications, vol. XI, New York, 1930 (referred to hereafter as Colloquium), pp. 10-12.

$\dagger$ For an account of this formula see D. Jackson, On approximation by trigonometric sums and polynomials, these Transactions, vol. 13 (1912), pp. 491-515; pp. 496-500. 
where $\Phi$ is given by a somewhat complicated formula when written out at full length, but has the essential properties that it depends on $x$ and $\xi$ only through the difference $\xi-x$ and is of period $2 \pi$.

If we differentiate $I_{s}(x)$ with respect to $x$, and replace $\partial \Phi / \partial x$ by its equal $-\partial \Phi / \partial \xi$ and integrate by parts, we get

$$
I_{s}^{\prime}(x)=h_{s} \int_{-\pi}^{\pi} f^{\prime}(\xi) \Phi(\xi-x) d \xi
$$

which is the $I_{s}$-function for $f^{\prime}(x)$ corresponding to the value $m$. But $f^{\prime}(x)$ is a function whose $(m-1)$ th derivative $f^{(m)}(x)$ satisfies a Lipschitz condition. Hence there will exist some constant $K_{m}$ such that

$$
\left|f^{\prime}(x)-I_{s}^{\prime}(x)\right| \leqq \frac{K_{m} \lambda}{n^{m}}
$$

Likewise we can obtain the inequalities

$$
\left|f^{\prime \prime}(x)-I_{s}^{\prime \prime}(x)\right| \leqq \frac{K_{m-1} \lambda}{n^{m-1}}, \ldots,\left|f^{(m)}(x)-I_{s}^{(m)}(x)\right| \leqq \frac{K_{1} \lambda}{n},
$$

where $K_{m-1}, \cdots, K_{1}$ are constants independent of $n$. If $A$ stands f., the greatest of the numbers $K_{m+1}, \cdots, K_{1}$, then

$$
\left|f^{(k)}(x)-I_{s}^{(k)}(x)\right| \leqq \frac{A}{n} \text { for } k=0,1, \cdots, m .
$$

Thus the function $I_{s}(x)$, with $s$ properly adjusted to correspond to $n$, can be made to serve the purpose of the $T_{n}(x)$ demanded by the theorem.

The corresponding theorem for polynomial approximation we shall call

THEOREM D. If, in addition to the hypotheses of Theorem B, it is assumed that $f^{(m)}(x)$ satisfies a Lipschitz condition on the interval $a \leqq x \leqq b$, then for each positive integral value of $n$ there exists a polynomial $P_{n}(x)$ of the nth degree such that

$$
\left|f^{(k)}(x)-P_{n}^{(k)}(x)\right| \leqq \frac{B}{n} \text { for } k=0,1, \cdots, m,
$$

and for all values of $x$ on the interval, where $B$ is a constant independent of $n$.

In the proof of Theorem $B$ the polynomial $Q_{1}(x)$ can be chosen so that $\left|f^{(m)}(x)-Q_{1}(x)\right|$ does not exceed a constant multiple of $1 / n,{ }^{*}$ and then

* Colloquium, pp. 13-14. 
the error in the approximation of each of the lower derivatives does not exceed this quantity multiplied by a factor independent of $n$.

It is worthy of special note that Theorems $\mathrm{C}$ and $\mathrm{D}$ can be generalized further. Thus if $f^{(m+\alpha)}(x)$ satisfies a Lipschitz condition, $\alpha$ being a positive integer, it can be shown by appropriate modification of the reasoning that the error in either the trigonometric or polynomial case does not exceed a quantity of the order of $1 / n^{1+\alpha}$.

For the treatment of polynomial convergence certain further results related to Theorems $\mathrm{B}$ and $\mathrm{D}$ will be required.

THEOREM E. If, in addition to the hypotheses of Theorem $\mathrm{B}$, it is assumed that $f(x)$ satisfies the $m$ linearly independent two-point boundary conditions

$$
U_{i}(f)=h_{i} \quad(i=1,2, \cdots, m),
$$

then for any positive number $\epsilon$ there exists a polynomial $P_{n}(x)$ of some degree $n$ such that $U_{i}\left(P_{n}\right)=h_{i}, i=1,2, \cdots, m$, and $\left|f^{(k)}(x)-P_{n}^{(k)}(x)\right| \leqq \epsilon$ for $k=0$, $1,2, \cdots, m$ and for all values of $x$ on the interval $(a, b)$.

Let $\epsilon^{\prime}$ be any positive number. Then, by Theorem B, there exists a polynomial $p_{n}(x)$ of some degree $n$ such that $\left|f^{(k)}(x)-p_{n}^{(k)}(x)\right| \leqq \epsilon^{\prime}$ for $k=0,1$, $2, \cdots, m$ and for $a \leqq x \leqq b$. Let the quantities $g_{1}, g_{2}, \cdots, g_{m}$ be defined by the equations $U_{i}\left(p_{n}\right)=h_{i}-g_{i}, i=1,2, \cdots, m$. Then it is clear that

$$
\left|g_{i}\right|=\left|U_{i}(f)-U_{i}\left(p_{n}\right)\right|=\left|U_{i}\left(f-p_{n}\right)\right| \leqq C \epsilon^{\prime} \quad(i=1,2, \cdots, m),
$$

where $C$ is a constant independent of $n$.

Let $q(x)$ denote a polynomial of the $(2 m-1)$ th degree, the coefficients of which are determined as follows. Set up the $2 m$ equations

$$
U_{1}(q)=g_{1}, \cdots, U_{m}(q)=g_{m}, U_{m+1}(q)=0, \cdots, U_{2 m}(q)=0,
$$

in which $U_{m+1}, \cdots, U_{2 m}$ are any linear combinations of the $2 m$ arguments $q(a), q^{\prime}(a), \cdots, q^{(m-1)}(a), q(b), \cdots, q^{(m-1)}(b)$, so chosen that all $2 m$ of the $U$ 's are linearly independent. Since the $U$ 's are linearly independent, the $2 m$ equations in the $2 m$ arguments will have a unique solution, and so will determine uniquely the values of these quantities. Hence if $q(x)$ has the form

$$
\begin{aligned}
q(x) & =A_{0}+A_{1}(x-a)+A_{2}(x-a)^{2}+\cdots+A_{m-1}(x-a)^{m-1} \\
& +A_{m}(x-a)^{m}+A_{m+1}(x-a)^{m}(x-b)+\cdots+A_{2 m-1}(x-a)^{m}(x-b)^{m-1},
\end{aligned}
$$

the coefficients $A_{0}, \cdots, A_{2 m-1}$ can be determined successively to fit the values $q(a), \cdots, q^{(m-1)}(a), q(b), \cdots, q^{(m-1)}(b)$.

With this definition of the polynomial $q(x)$ it is not difficult to see that an upper bound placed on the absolute values of the $g$ 's in the $2 m$ equations 
above sets an upper bound for $|q(a)|, \cdots,\left|q^{(m-1)}(b)\right|$, and this in turn sets an upper bound for the absolute values of the coefficients in $q(x)$ and so for the absolute values of $q(x)$ itself and all its derivatives throughout the interval. Hence, since $C \epsilon^{\prime}$ is an upper bound for the $g$ 's, we can write $\left|q^{(k)}(x)\right| \leqq C^{\prime} \epsilon^{\prime}$ for $k=0,1,2, \cdots, m$ and $a \leqq x \leqq b$, where $C^{\prime}$ is a constant independent of $n$.

Let us now consider the function $P_{n}(x)=p_{n}(x)+q(x)$. This is a polynomial of the $n$th degree (if $n \geqq 2 m-1^{*}$ ) which satisfies the $m$ boundary equations $U_{i}\left(P_{n}\right)=h_{i}$, since $p_{n}(x)$ and $q(x)$ satisfy the conditions $U_{i}\left(p_{n}\right)$ $=h_{i}-g_{i}, U_{i}(q)=g_{i}$ respectively, and furthermore,

$$
\left|f^{(k)}(x)-P_{n}^{(k)}(x)\right|=\left|f^{(k)}(x)-p_{n}^{(k)}(x)-q^{(k)}(x)\right| \leqq\left(1+C^{\prime}\right) \epsilon^{\prime},
$$

for $k=0,1,2, \cdots, m$, and $a \leqq x \leqq b$. If $\epsilon$ is arbitrary, and if $\epsilon^{\prime}$ is taken equal to $\epsilon /\left(1+C^{\prime}\right)$, the polynomial $P_{n}(x)$ will then fulfill the requirements of the theorem.

The above proof shows in substance that if $f(x)$ satisfies the boundary conditions and if polynomials $p_{n}(x)$ can be determined so that $\mid f^{(k)}(x)$ $-p_{n}^{(k)}(x) \mid \leqq \epsilon_{n}$ then there exist polynomials $P_{n}(x)$ satisfying the boundary conditions and such that $\left|f^{(k)}(x)-P_{n}^{(k)}(x)\right| \leqq\left(1+C^{\prime}\right) \epsilon_{n}$, where $C^{\prime}$ is a constant independent of $n$. Combined with Theorem $\mathrm{D}$, this yields

TheOREM F. Under the hypotheses of Theorem $\mathrm{E}$ and the additional hypothesis that $f^{(m)}(x)$ satisfies a Lipschitz condition on the interval, there exists for each positive integral value of $n \geqq 2 m-1$ a polynomial $P_{n}(x)$ of the nth degree such that $U_{i}\left(P_{n}\right)=h_{i}, i=1,2, \cdots, m$, and such that

$$
\left|f^{(k)}(x)-P_{n}^{(k)}(x)\right| \leqq D / n \quad(k=0,1, \cdots, m),
$$

for all values of $x$ on the interval, where $D$ is a constant independent of $n$.

The generalization of Theorem $\mathrm{F}$ when $f(x)$ exhibits higher properties of continuity is obvious.

4. Convergence in the trigonometric case when $r \geqq 1$. In this section we shall discuss the questions of convergence and degree of approximation relative to a periodic differential system for which the minimizing function is a trigonometric sum defined for some value of $r \geqq 1$.

Suppose we have given the differential system

* The form of statement of the conclusion is not affected by the fact that a finite number of values of $n$ have to be set aside as exceptional in the course of the proof, to insure the possibility of satisfying the boundary conditions. 


$$
\begin{aligned}
L(y) & \equiv \frac{d^{m} y}{d x^{m}}+Q_{1}(x) \frac{d^{m-1} y}{d x^{m-1}}+\cdots+Q_{m}(x) y=R(x), \\
U_{i}(y) & \equiv y^{(i-1)}(0)-y^{(i-1)}(2 \pi)=0 \quad(i=1,2, \cdots, m),
\end{aligned}
$$

in which the functions $Q_{1}(x), \cdots, Q_{m}(x), R(x)$ are continuous and periodic with the period $2 \pi$, and suppose further that the reduced system

$$
\begin{aligned}
L(y) & =0, \\
U_{i}(y) & =0 \quad(i=1,2, \cdots, m)
\end{aligned}
$$

is incompatible, so that (1) has a unique solution $y(x)$. Then $y(x)$ and its first $m$ derivatives will necessarily be continuous and periodic with the period $2 \pi$.

As functions $\phi_{i}(x)$ we shall use the periodic trigonometric functions $1, \cos x, \sin x, \cos 2 x, \sin 2 x, \cdots$. The minimizing function of order $2 n+1$ is then a trigonometric sum $T_{n}(x)$ of order $n$,

$$
\begin{aligned}
y_{2 n+1}(x)=T_{n}(x)=a_{0} & +a_{1} \cos x+\cdots+a_{n} \cos n x \\
& +b_{1} \sin x+\cdots+b_{n} \sin n x,
\end{aligned}
$$

in which the coefficients are so determined that the integral

$$
\int_{0}^{2 \pi}\left|L\left(T_{n}\right)-R\right| r d x=\int_{0}^{2 \pi}\left|L\left(y-T_{n}\right)\right| r d x, \quad r \geqq 1,
$$

is a minimum. The function $T_{n}(x)$ may be described as "the minimizing trigonometric sum of order $n$ for $y(x)$ corresponding to the exponent $r . "$

The function $y(x)$ satisfies the hypotheses of Theorem A, and hence, if $\epsilon$ is any positive number, there exists a trigonometric sum $t_{n}(x)$ of some order $n$ such that $\left|y^{(k)}(x)-t_{n}^{(k)}(x)\right| \leqq \epsilon$, for $k=0,1, \cdots, m$. Let $F(x)=y(x)-t_{n}(x)$ and let $\pi_{n}(x)$ be the minimizing sum of order $n$ for $F(x)$. Then the integral

$$
\gamma=\int_{0}^{2 \pi}\left|L\left(F-\pi_{n}\right)\right|^{r} d x
$$

is a minimum, and therefore, since zero is itself a trigonometric sum of order $n$ (in which the coefficients are all zero), we can write

But

$$
\gamma \leqq \int_{0}^{2 \pi}|L(F)| r d x
$$

$$
|L(F)|=\left|F^{(m)}+Q_{1} F^{(m-1)}+\cdots+Q_{m} F\right| \leqq(m+1) M \epsilon,
$$

where $M$ is an upper bound for the bounded functions $1,\left|Q_{1}\right|, \ldots,\left|Q_{m}\right|$. Hence 


$$
\gamma \leqq 2 \pi\{(m+1) M \epsilon\}
$$

Let $u(x)=F(x)-\pi_{n}(x)$ and let $Z(x)=L(u)$. Then $u(x)$ satisfies the differential system

$$
\begin{array}{rlrl}
L(u) & =Z(x), \\
U_{i}(u) & =0 & (i=1,2, \cdots, m),
\end{array}
$$

and indeed is the unique solution, since (2) is incompatible. Let $G(x, \xi)$ be the Green's function associated with (2).* Then

Likewise

$$
u(x)=\int_{0}^{2 \pi} G(x, \xi) Z(\xi) d \xi .
$$

$$
\begin{gathered}
u^{\prime}(x)=\int_{0}^{2 \pi} \frac{\partial}{\partial x} G(x, \xi) Z(\xi) d \xi \\
\text {. . . . . . . . . . } \\
u^{(m-1)}(x)=\int_{0}^{2 \pi} \frac{\partial^{m-1}}{\partial x^{m-1}} G(x, \xi) Z(\xi) d \xi .
\end{gathered}
$$

The differentiation under the integral sign might be questioned in the case of the last equation on the ground that $\left(\partial^{m-1} / \partial x^{m-1}\right) G(x, \xi)$ is discontinuous on the diagonal $x=\xi$. By considering separately the integrals from .0 to $x$ and from $x$ to $2 \pi$, however, it can be shown that the formula is valid in this case also. $\dagger$

Let $\bar{G}$ be an upper bound for the bounded functions $|G(x, \xi)|, \cdots$, $\left|\left(\partial^{m-1} / \partial x^{m-1}\right) G(x, \xi)\right|$. Then

$$
\left|u^{(k)}(x)\right| \leqq \bar{G} \int_{0}^{2 \pi}|Z(\xi)| d \xi \quad(k=0,1, \cdots, m-1) .
$$

We make use of Hölder's inequality in the form which states that if $\phi(x) \geqq 0$ and $r$ is any real number $\geqq 1$ then

$$
\int_{a}^{b} \phi(x) d x \leqq(b-a)^{(r-1) / r}\left[\int_{a}^{b}\{\phi(x)\} r d x\right]^{1 / r}
$$

In the present case this gives $\ddagger$

$$
\left|u^{(k)}(x)\right| \leqq(2 \pi)^{(r-1) / r} \bar{G}\left[\int_{0}^{2 \pi}|Z(\xi)| r d \xi\right]^{1 / r}=(2 \pi)^{(r-1) / r} \bar{G} \gamma^{1 / r}
$$

* The writer is indebted to Professor D. V. Widder for a valuable suggestion in connection with this stage of the work.

$\dagger$ See, for example, Bocher, Leçons sur les Methodes de Sturm, Paris, 1917, pp. 102-103.

$\ddagger$ It is at this point that we use the hypothesis in the definition of the minimizing sums that $r \geqq 1$. 
and by (3), if $N$ denotes the quantity $2 \pi \bar{G} M$, independent of $n$,

$$
\left|u^{(k)}(x)\right| \leqq N \epsilon \quad(k=0,1, \cdots, m-1) .
$$

But since $\pi_{n}(x)$ and $T_{n}(x)$ are the respective minimizing trigonometric sums for $F(x)$ and $y(x)$, two functions whose difference is the trigonometric sum $t_{n}(x)$, it is readily seen that $\pi_{n}(x)$ is identical with $T_{n}(x)-t_{n}(x)$. Hence

$$
u(x)=F(x)-\pi_{n}(x)=y(x)-T_{n}(x),
$$

and consequently

$$
\left|y^{(k)}(x)-T_{n}^{(k)}(x)\right| \leqq N \epsilon \quad(k=0,1, \cdots, m-1) .
$$

This is true for any value of $n$ for which a trigonometric sum $t_{n}(x)$ of the $n$th order exists, satisfying the relations $\left|y^{(k)}(x)-t_{n}^{(k)}(x)\right| \leqq \epsilon, k=0,1, \cdots$, $m-1$, and so, in the case of any specified positive $\epsilon$, is true for all values of $n$ from a certain point on. Thus it is possible to state

THEOREM I. If the periodic system (1) has a unique solution $y(x)$, the minimizing trigonometric sum $T_{n}(x)$ of order $n$ corresponding to any given real number $r \geqq 1$ converges uniformly to the value of $y(x)$ as $n$ becomes infinite, and furthermore, the first $(m-1)$ derivatives of $T_{n}(x)$ converge uniformly to the respective derivatives of $y(x)$.

If $y^{(m)}(x)$ satisfies a Lipschitz condition, which will necessarily be the case if the functions $Q_{1}(x), \cdots, Q_{m}(x), R(x)$ satisfy such a condition, then, by Theorem $C$, sums $t_{n}(x)$ can be determined so that $\left|y^{(k)}(x)-t_{n}^{(k)}(x)\right|$ has an upper bound of the order of $1 / n$. Hence we can state

THEOREM II. If, in addition to the hypotheses of Theorem I, it is assumed that $y^{(m)}(x)$ satisfies a Lipschitz condition, then for all positive integral values of $n$

$$
\left|y^{(k)}(x)-T_{n}^{(k)}(x)\right| \leqq \frac{E}{n} \quad(k=0,1, \cdots, m-1),
$$

where $E$ is a constant independent of $n$.

5. Convergence in the polynomial case when $r \geqq 1$. Let us consider again the differential system with non-homogeneous boundary conditions described in $\$ 1$. It has the form

$$
\begin{aligned}
L(y) & =R(x), & \\
U_{i}(y) & =h_{i} & (i=1,2, \cdots, m) .
\end{aligned}
$$

The coefficients in the differential equation are defined and continuous on $a \leqq x \leqq b$, the boundary conditions are linearly independent, and the reduced system 


$$
\begin{aligned}
L(y) & =0, \\
U_{\text {i }}(y) & =0
\end{aligned} \quad(i=1,2, \cdots, m)
$$

is incompatible. If $y(x)$ is the solution, it is unique, and it and its first $m$ derivatives are continuous on the interval.

As functions $\phi_{i}(x)$ we shall use $1, x, x^{2}, \cdots$. The minimizing function of order $n+1$ corresponding to a given value of $r \geqq 1$ is then a polynomial $P_{n}(x)$ of the $n$th degree:

$$
y_{n+1}=P_{n}(x)=c_{0}+c_{1} x+c_{2} x^{2}+\cdots+c_{n} x^{n}
$$

which satisfies the $m$ boundary equations $U_{i}\left(P_{n}\right)=h_{i}$, and is such that the integral

$$
\int_{a}^{b}\left|L\left(y-P_{n}\right)\right| r d x, r \geqq 1,
$$

is a minimum. The function $P_{n}(x)$ may be called "the minimizing polynomial of the $n$th degree for $y(x)$ corresponding to the exponent $r . "$

The function $y(x)$ satisfies the hypotheses of Theorem $\mathrm{E}$, and hence if $\epsilon$ is any positive number, there exists a polynomial $p_{n}(x)$ of some degree $n$ which satisfies the boundary conditions $U_{i}\left(p_{n}\right)=h_{i}, i=1,2, \cdots, m$, and is such that $\left|y^{(k)}(x)-p_{n}^{(k)}(x)\right| \leqq \epsilon$ for $k=0,1, \cdots, m$. Let $F(x)=y(x)-p_{n}(x)$. Then $F(x)$ satisfies the $m$ homogeneous boundary conditions $U_{i}(F)=0$, $i=1,2, \cdots, m$. Let $\pi_{n}(x)$ be the minimizing polynomial of the $n$th degree for $F(x)$ which satisfies the same homogeneous equations $U_{i}\left(\pi_{n}\right)=0$. Then the integral

$$
\gamma=\int_{a}^{b}\left|L\left(F-\pi_{n}\right)\right|^{r} d x
$$

is a minimum for polynomials of this type.

But zero is itself a polynomial of the $n$th degree, in which the coefficients are all zero, and it satisfies the homogeneous boundary equations. Hence

$$
\gamma \leqq \int_{a}^{b}|L(F)|^{r} d x
$$

and consequently, if $M$ is an upper bound of the bounded functions 1, $\left|Q_{1}\right|, \cdots,\left|Q_{m}\right|$,

$$
\gamma \leqq(b-a)\{(m+1) M \epsilon\} r .
$$

Let $u(x)=F(x)-\pi_{n}(x)$ and let $Z(x)=L(u)$. Then $u(x)$ is the unique solution of the differential system 


$$
\begin{array}{rlrl}
L(u) & =Z(x), \\
U_{i}(u) & =0 & (i=1,2, \cdots, m),
\end{array}
$$

and therefore, if $G(x, \xi)$ is the Green's function associated with the reduced ystem (5),

$$
u^{(k)}(x)=\int_{a}^{b} \frac{\partial^{k}}{\partial x^{k}} G(x, \xi) Z(\xi) d \xi \quad(k=0,1, \cdots, m-1) .
$$

Without detailed repetition of the argument used in the last section it is clear that $u(x)=y(x)-P_{n}(x)$, and that there is a number $N$, independent of $n$, such that

$$
\left|y^{(k)}(x)-P_{n}^{(k)}(x)\right| \leqq N \epsilon \quad(k=0,1, \cdots, m-1),
$$

for all values of $x$ on $(a, b)$. To cover the conclusions in this case we can state the following two theorems:

Theorem III. If the differential system (4) has a unique solution $y(x)$, then the minimizing polynomial $P_{n}(x)$ of the nth degree, corresponding to a given value of $r \geqq 1$, converges uniformly on the interval $a \leqq x \leqq b$ to the value of $y(x)$ as $n$ becomes infinite, and the first $m-1$ derivatives of $P_{n}(x)$ converge uniformly to the respective derivatives of $y(x)$.

THEOREM IV. If, in addition to the hypotheses of Theorem III, it is assumed that $y^{(m)}(x)$ satisfies a Lipschitz condition on the interval, then the errors $\left|y^{(k)} \cdot(x)-P_{n}{ }^{(k)}(x)\right|$ have an upper bound of the order of $1 / n$.

6. Preliminary theorems for the treatment of convergence when $r<1$. The foregoing proofs of convergence were based on a direct application of Hölder's inequality, a relation which holds only when $r \geqq 1$. Consequently when the minimizing sum is defined for a value of $r<1$ a different method must be used. In this section we shall derive two theorems in preparation for the discussion of this case; one is an extension of Bernstein's theorem on the derivative of a trigonometric sum, and the other an extension of Markoff's heorem on the derivative of a polynomial.

TheOREM G. If $T_{n}(x)$ is an arbitrary trigonometric sum of the nth order, and if

$$
\begin{aligned}
L(y) & =R(x), \\
U_{i}(y) & \equiv y^{(i-1)}(0)-y^{(i-1)}(2 \pi)=0 \quad(i=1,2, \cdots, m),
\end{aligned}
$$

is the differential system (1) described in $\$ 4$, of which the reduced system is incompatible, and if $\delta=\max \left|L\left(T_{n}\right)\right|$, then 
(a)

$$
\text { . }\left|T_{n}^{(k)}(x)\right| \leqq A \delta \quad(k=0,1, \cdots, m),
$$

and if $Q_{1}(x), \cdots, Q_{m}(x)$ have bounded first derivatives,

$$
\left|\frac{d}{d x} L\left(T_{n}\right)\right| \leqq B n \delta,
$$

where $A$ and $B$ are constants independent of $n$ and independent of the coefficients in $T_{n}(x)$.

Let $Z(x)=L\left(T_{n}\right)$. Then $T_{n}(x)$, being periodic with the period $2 \pi$, will satisfy the differential system

$$
L(y)=Z(x), \quad U_{i}(y)=0 \quad(i=1,2, \cdots, m) .
$$

But the reduced system is incompatible, and so, if $G(x, \xi)$ is its Green's function, we can write

$$
T_{n}^{(k)}(x)=\int_{0}^{2 \pi} \frac{\partial^{k}}{\partial x^{k}} G(x, \xi) Z(\xi) d \xi \quad(k=0,1, \cdots, m-1) .
$$

Let $\bar{G}$ be an upper bound of the bounded functions $|G(x, \xi)|, \cdots$, $\left|\left(\partial^{m-1} / \partial x^{m-1}\right) G(x, \xi)\right|$. Then since $\delta$ is an upper bound of $|Z(\xi)|$,

$$
\left|T_{n}^{(k)}(x)\right| \leqq 2 \pi \bar{G} \delta
$$

for $k=0,1, \cdots, m-1$. By virtue of these relations and the identity $T_{n}^{(m)}(x)=L\left(T_{n}\right)-Q_{1} T_{n}^{(m-1)}-\cdots-Q_{m} T_{n}$ it follows that $\left|T_{n}^{(m)}(x)\right|$ also has an upper bound of the same form:

$$
\left|T_{n}^{(m)}(x)\right| \leqq \delta+m M(2 \pi \bar{G} \delta)=(1+2 \pi m M \bar{G}) \delta,
$$

where $M$ is an upper bound of $1,\left|Q_{1}\right|, \ldots,\left|Q_{m}\right|$. By letting $A$ stand for the greater of the two numbers $2 \pi \bar{G},(1+2 \pi m M \bar{G})$ we obtain conclusion (a).

The function $T_{n}^{(m)}(x)$ is a trigonometric sum of the $n$th order of which $A \delta$ is an upper bound. Hence, by Bernstein's theorem on the derivative of a trigonometric sum,

$$
\left|T_{n}^{(m+1)}(x)\right| \leqq A n \delta
$$

and therefore,

$$
\begin{aligned}
\left|\frac{d}{d x} L\left(T_{n}\right)\right|= & \mid T_{n}^{(m+1)}+\left(Q_{1} T_{n}^{(m)}+\cdots+Q_{m} T_{n}^{\prime}\right)+\left(Q_{1}^{\prime} T_{n}^{(m-1)}+\cdots\right. \\
& \left.+Q_{m}^{\prime} T_{n}\right) \mid \\
\leqq & A n \delta+m M A \delta+m M^{\prime} A \delta \leqq B n \delta,
\end{aligned}
$$


where $M^{\prime}$ is an upper bound of $\left|Q_{1}^{\prime}\right|, \cdots,\left|Q_{m}^{\prime}\right|$ and $B=A\left(1+m M+m M^{\prime}\right)$. This is conclusion (b). (It is readily seen from the details of the calculation, or can evidently be assumed outright without affecting the truth of the conclusion, that $B \geqq 1$.)

TheOREM H. If

$$
\begin{aligned}
L(y) & =R(x), & \\
U_{i}(y) & =h_{i} & (i=1,2, \cdots, m),
\end{aligned}
$$

is the differential system (4) with general non-homogeneous two-point boundary conditions, of which the reduced system is incompatible, and if $P_{n}(x)$ is any polynomial of the nth degree which satisfies the homogeneous boundary equations

$$
U_{i}\left(P_{n}\right)=0 \quad(i=1,2, \cdots, m),
$$

and if $\delta=\max \left|L\left(P_{n}\right)\right|$ on $a \leqq x \leqq b$, then

$$
\left|P_{n}^{(k)}(x)\right| \leqq C \delta \quad(k=0,1, \cdots, m),
$$

and if $Q_{1}(x), \cdots, Q_{m}(x)$ have bounded first derivatives,

$$
\left|\frac{d}{d x} L\left(P_{n}\right)\right| \leqq D n^{2} \delta
$$

for all values of $x$ on $(a, b)$, where $C$ and $D$ are constants independent of $n$ and independent of the coefficients in $P_{n}(x)$.

The method of proof here is practically the same as in Theorem G, except that Markoff's theorem* is used instead of Bernstein's theorem in obtaining conclusion (b).

7. Convergence when $r<1$. In this section the convergence problem when $r<1$ will be discussed in detail for the trigonometric case and only the results stated for the polynomial case. It will be seen that the method used here $\dagger$ is not restricted in application to the cases when $r<1$, but is generally applicable when $r$ is any positive real number. Moreover, although the bound which it assigns to the errors is not as good as that obtained by the first method under given hypotheses when $r \geqq 1$, it proves the convergence of $m$ derivatives, as compared with $m-1$ in the earlier treatment.

* See, for example, D. Jackson, these Transactions, vol. 22, loc. cit., p. 163; M. Riesz, Eine trigonometrische Interpolationsformel und einige Ungleichungen für Polynome, Jahresbericht der Deutschen Mathematiker-Vereinigung, vol. 23 (1914), pp. 354-368.

$\dagger$ The general scheme was suggested by convergence proofs given in other connections by $D$. Jackson; see, for example, On the convergence of certain trigonometric and polynomial approximations, these Transactions, vol. 22 (1921), pp. 158-166; Colloquium, Chapter III. 
Let it be assumed that the periodic system

$$
\begin{aligned}
L(y) & =R(x), \\
U_{i}(y) & \equiv y^{(i-1)}(0)-y^{(i-1)}(2 \pi)=0 \quad(i=1,2, \cdots, m),
\end{aligned}
$$

has the unique solution $y(x)$, and that the coefficients in the differential equation are provided with the derivatives called for. Let $t_{n}(x)$ be an arbitrary trigonometric sum of the $n$th order, and let $\epsilon$ be a number such that

$$
\left|y^{(k)}(x)-t_{n}^{(k)}(x)\right| \leqq \epsilon \quad(k=0,1, \cdots, m)
$$

for all values of $x$. Let $F(x)=y(x)-t_{n}(x)$, and let $\pi_{n}(x)$ be a minimizing sum of order $n$ for $F(x)$. Then the integral

$$
\gamma=\int_{0}^{2 \pi}\left|L\left(F-\pi_{n}\right)\right| r d x
$$

is a minimum, and since zero is itself a trigonometric sum of the $n$th order,

$$
\gamma \leqq \int_{0}^{2 \pi}|L(F)|^{r} d x \leqq 2 \pi\{(m+1) M \epsilon\}^{m},
$$

where $M$ is again an upper bound for $1,\left|Q_{1}\right|, \cdots,\left|Q_{m}\right|$.

Let $x_{1}$ be a point of the interval $(0,2 \pi)$ at which $\left|L\left(\pi_{n}\right)\right|$ takes on its maximum value $\delta$. Then, by virtue of the mean value theorem and conclusion (b) of Theorem G,

$$
\left|L\left(\pi_{n}(x)\right)-L\left(\pi_{n}\left(x_{1}\right)\right)\right| \leqq\left|x-x_{1}\right| B n \delta .
$$

Therefore for values of $x$ in the interval $\left|x-x_{1}\right| \leqq 1 /[2 B n]$,

$$
\left|L\left(\pi_{n}\right)\right| \geqq \delta / 2 \text {. }
$$

Assume for the moment that $\epsilon<\delta /[4(1+m) M]$. Then

$$
|L(F)|=\left|F^{(m)}+Q_{1} F^{(m-1)}+\cdots+Q_{m} F\right| \leqq(m+1) M \epsilon<\delta / 4
$$

and therefore, when $x$ belongs to the interval $\left|x-x_{1}\right| \leqq 1 /[2 B n], \mid L\left(\pi_{n}\right)$ $-L(F) \mid \geqq \delta / 4$, and hence

$$
\gamma \geqq \int_{x_{1}-1 /(2 B n)}^{x_{1}+1 /(2 B n)}\left|L\left(\pi_{n}\right)-L(F)\right| r d x \geqq \frac{1}{B n}\left(\frac{\delta}{4}\right)^{r} .
$$

Combining this with (7) we get

$$
\delta \leqq 4(2 \pi B)^{1 / r}(m+1) M n^{1 / r} \epsilon .
$$

But this relation is true also when $\epsilon \geqq \delta /[4(m+1) M]$ and so it holds universally. 
Consider the errors

$$
\left|F^{(k)}(x)-\pi_{n}^{(k)}(x)\right| \quad(k=0,1, \cdots, m) .
$$

By virtue of conclusion (a) of Theorem $\mathrm{G}$ and relation (8),

$$
\begin{aligned}
\left|F^{(k)}(x)-\pi_{n}{ }^{(k)}(x)\right| & \leqq \epsilon+A \delta \leqq \epsilon+4 A(2 \pi B)^{1 / r}(m+1) M n^{1 / r} \epsilon \\
& \leqq E n^{1 / r} \epsilon,
\end{aligned}
$$

where $E$ is a constant independent of $n$. But, since $F(x)$ and $y(x)$ differ only by the terms of the trigonometric sum $t_{n}(x)$, their respective minimizing sums will differ only by $t_{n}(x)$. Hence if $T_{n}(x)$ is the minimizing sum for $y(x)$,

$$
\left|y^{(k)}(x)-T_{n}^{(k)}(x)\right|=\left|F^{(k)}(x)-\pi_{n}^{(k)}(x)\right| \leqq E n^{1 / \tau}
$$

for $k=0,1, \cdots, m$ and for all values of $x$.

The questions of convergence and degree of approximation are thus directly connected with the approximations in (6). Any hypotheses that will make it possible to define sums $t_{n}(x)$ so that $n^{1 / r} \epsilon$ approaches zero as $n$ becomes infinite will insure the convergence of $T_{n}(x)$ and its first $m$ derivatives to the respective values of $y(x)$ and its derivatives. For example, when $r=\frac{1}{2}$ it is sufficient to assume that $y^{(m+2)}(x)$ satisfies a Lipschitz condition, for then $\epsilon$ can be made to have the order of $1 / n^{3}$ and $n^{1 / r} \epsilon$ the order of $1 / n$.

The conclusion, and the result obtained by the same method for the case of polynomial approximation, are expressed by the following two theorems:

THEOREM V. If the periodic system

$$
\begin{aligned}
L(y) & =R(x), \\
U_{i}(y) & =0
\end{aligned} \quad(i=1,2, \cdots, m),
$$

has a unique solution $y(x)$ of such regularity that it and its first $m$ derivatives can be simultaneously approximated by a trigonometric sum of the nth order and its corresponding derivatives with an upper bound of error $\epsilon$ satisfying the condition that

$$
\lim _{n \rightarrow \infty} n^{1 / r_{\epsilon}}=0
$$

and if the coefficients in $L(y)$ have bounded first derivatives, then the minimizing trigonometric sum and its first $m$ derivatives converge uniformly to $y(x)$ and its corresponding derivatives respectively as $n$ becomes infinite.

Theorem VI. If the system

$$
\begin{aligned}
L(y) & =R(x), \\
U_{i}(y) & =h_{i}
\end{aligned} \quad(i=1,2, \cdots, m),
$$


with general non-homogeneous two-point boundary conditions, has a unique solution $y(x)$ of such regularity that it and its first $m$ derivatives can be simultaneously approximated on the interval $(a, b)$ by means of a polynomial of the nth degree, satisfying the boundary conditions, and its derivatives, with an upper bound of error $\epsilon$ for which

$$
\lim _{n \rightarrow \infty} n^{2 / r_{\epsilon}}=0
$$

and if the coefficients in $L(y)$ have bounded first derivatives, then the minimizing polynomial satisfying the boundary conditions, and its first $m$ derivatives, converge uniformly on the interval to the respective values of $y(x)$ and its corresponding derivatives as $n$ becomes infinite.

Mount Alirson University,

SACKVILLE, NeW BrUNSWICK 